\title{
Energy dependence of pion and kaon production in central $\mathrm{Pb}+\mathrm{Pb}$ collisions
}

S. V. Afanasiev, ${ }^{9}$ T. Anticic, ${ }^{20}$ D. Barna,${ }^{5}$ J. Bartke, ${ }^{7}$ R. A. Barton, ${ }^{3}$ M. Behler, ${ }^{15}$ L. Betev,${ }^{10}$ H. Białkowska, ${ }^{17}$ A. Billmeier, ${ }^{10}$ C. Blume, ${ }^{8}$ C. O. Blyth, ${ }^{3}$ B. Boimska, ${ }^{17}$ M. Botje, ${ }^{1}$ J. Bracinik,${ }^{4}$ R. Bramm, ${ }^{10}$ R. Brun, ${ }^{11}$ P. Bunčić $,{ }^{10}, 11$ V. Cerny, ${ }^{4}$ J. G. Cramer, ${ }^{19}$ P. Csató, ${ }^{5}$ P. Dinkelaker, ${ }^{10}$ V. Eckhardt, ${ }^{16}$ P. Filip, ${ }^{16}$ Z. Fodor,,${ }^{5}$ P. Foka ${ }^{8}$ P. Freund, ${ }^{16}$ V. Friese,${ }^{15}$ J. Gál, ${ }^{5}$ M. Gaździcki, ${ }^{10}$ G. Georgopoulos,${ }^{2}$ E. Gładysz, ${ }^{7}$ S. Hegyi,,${ }^{5}$ C. Höhne, ${ }^{15}$ G. Igo, ${ }^{14}$ P. G. Jones, ${ }^{3}$ K. Kadija,,${ }^{11,20}$

A. Karev, ${ }^{16}$ V. I. Kolesnikov, ${ }^{9}$ T. Kollegger, ${ }^{10}$ M. Kowalski, ${ }^{7}$ I. Kraus, ${ }^{8}$ M. Kreps,${ }^{4}$ M. van Leeuwen, ${ }^{1}$ P. Lévai, ${ }^{5}$ A. I. Malakhov, ${ }^{9}$ S. Margetis, ${ }^{13}$ C. Markert, ${ }^{8}$ B. W. Mayes, ${ }^{12}$ G. L. Melkumov, ${ }^{9}$ A. Mischke, ${ }^{8}$ J. Molnár,,${ }^{5}$ J. M. Nelson, ${ }^{3}$ G. Pálla, ${ }^{5}$ A. D. Panagiotou, ${ }^{2}$ K. Perl,${ }^{18}$ A. Petridis, ${ }^{2}$ M. Pikna, ${ }^{4}$ L. Pinsky, ${ }^{12}$ F. Pühlhofer, ${ }^{15}$ J. G. Reid,${ }^{19}$

R. Renfordt, ${ }^{10}$ W. Retyk, ${ }^{18}$ C. Roland, ${ }^{6}$ G. Roland, ${ }^{6}$ A. Rybicki, ${ }^{7}$ T. Sammer ${ }^{16}$ A. Sandoval,${ }^{8}$ H. Sann, ${ }^{8}$ N. Schmitz, ${ }^{16}$ P. Seyboth, ${ }^{16}$ F. Siklér, ${ }^{5}$ B. Sitar, ${ }^{4}$ E. Skrzypczak, ${ }^{18}$ G. T. A. Squier, ${ }^{3}$ R. Stock, ${ }^{10}$ H. Ströbele, ${ }^{10}$ T. Susa, ${ }^{20}$ I. Szentpétery, ${ }^{5}$ J. Sziklai, ${ }^{5}$ T. A. Trainor, ${ }^{19}$ D. Varga, ${ }^{5}$ M. Vassiliou, ${ }^{2}$ G. I. Veres, ${ }^{5}$ G. Vesztergombi, ${ }^{5}$ D. Vranić, ${ }^{8}$ A. Wetzler, ${ }^{10}$ C. Whitten ${ }^{14}$ I. K. Yoo, ${ }^{15}$ J. Zaranek, ${ }^{10}$ and J. Zimányi ${ }^{5}$

(The NA49 Collaboration)

${ }^{1}$ NIKHEF, Amsterdam, Netherlands

${ }^{2}$ Department of Physics, University of Athens, Athens, Greece

${ }^{3}$ Birmingham University, Birmingham, England

${ }^{4}$ Comenius University, Bratislava, Slovakia

${ }^{5}$ KFKI Research Institute for Particle and Nuclear Physics, Budapest, Hungary

${ }^{6}$ MIT, Cambridge, Massachusetts 02139-4307

${ }^{7}$ Institute of Nuclear Physics, Cracow, Poland

${ }^{8}$ Gesellschaft für Schwerionenforschung (GSI), Darmstadt, Germany

${ }^{9}$ Joint Institute for Nuclear Research, Dubna, Russia

${ }^{10}$ Fachbereich Physik der Universität, Frankfurt, Germany

${ }^{11}$ CERN, Geneva, Switzerland

${ }^{12}$ University of Houston, Houston, Texas 77204-5506

${ }^{13}$ Kent State University, Kent, Ohio 49292

${ }^{14}$ University of California at Los Angeles, Los Angeles, California 90024

${ }^{15}$ Fachbereich Physik der Universität, Marburg, Germany

${ }^{16}$ Max-Planck-Institut für Physik, Munich, Germany

${ }^{17}$ Institute for Nuclear Studies, Warsaw, Poland

${ }^{18}$ Institute for Experimental Physics, University of Warsaw, Warsaw, Poland

${ }^{19}$ Nuclear Physics Laboratory, University of Washington, Seattle, Washington 98195

${ }^{20}$ Rudjer Boskovic Institute, Zagreb, Croatia

(Received 6 May 2002; published 27 November 2002)

\begin{abstract}
Measurements of charged pion and kaon production in central $\mathrm{Pb}+\mathrm{Pb}$ collisions at 40,80 , and $158 \mathrm{AGeV}$ are presented. These are compared with data at lower and higher energies as well as with results from $p+p$ interactions. The mean pion multiplicity per wounded nucleon increases approximately linearly with $s_{N N}^{1 / 4}$ with a change of slope starting in the region 15-40 A GeV. The change from pion suppression with respect to $p$ $+p$ interactions, as observed at low collision energies, to pion enhancement at high energies occurs at about $40 \mathrm{~A} \mathrm{GeV}$. A nonmonotonic energy dependence of the ratio of $K^{+}$to $\pi^{+}$yields is observed, with a maximum close to $40 \mathrm{~A} \mathrm{GeV}$ and an indication of a nearly constant value at higher energies. The measured dependences may be related to an increase of the entropy production and a decrease of the strangeness to entropy ratio in central $\mathrm{Pb}+\mathrm{Pb}$ collisions in the low SPS energy range, which is consistent with the hypothesis that a transient state of deconfined matter is created above these energies. Other interpretations of the data are also discussed.
\end{abstract}

DOI: 10.1103/PhysRevC.66.054902

PACS number(s): 25.75. $-\mathrm{q}$

\section{INTRODUCTION}

The primary purpose of the heavy ion program at the CERN SPS is the search for a transient deconfined state of strongly interacting matter during the early stage of nucleusnucleus collisions [1]. When a sufficiently high initial energy density is reached, the formation of such a state of quasifree quarks and gluons, the quark gluon plasma (QGP), is expected. A key problem is the identification of experimental signatures of QGP creation [2]. Numerous proposals were discussed in the past [3], but the significance of these signals has come under renewed scrutiny. A possible, promising strategy is a study of the energy dependence of pion and strangeness yields. It was suggested [4-6] that the transition may lead to anomalies in this dependence: a steepening of the increase of the pion yield and a nonmonotonic behavior of the strangeness to pion ratio. The spacetime evolution of the created fireball [7] and the event-by-event fluctuations [8] may also be sensitive to crossing the transition region.

First experimental results from $\mathrm{Pb}+\mathrm{Pb}(\mathrm{Au}+\mathrm{Au})$ colli- 


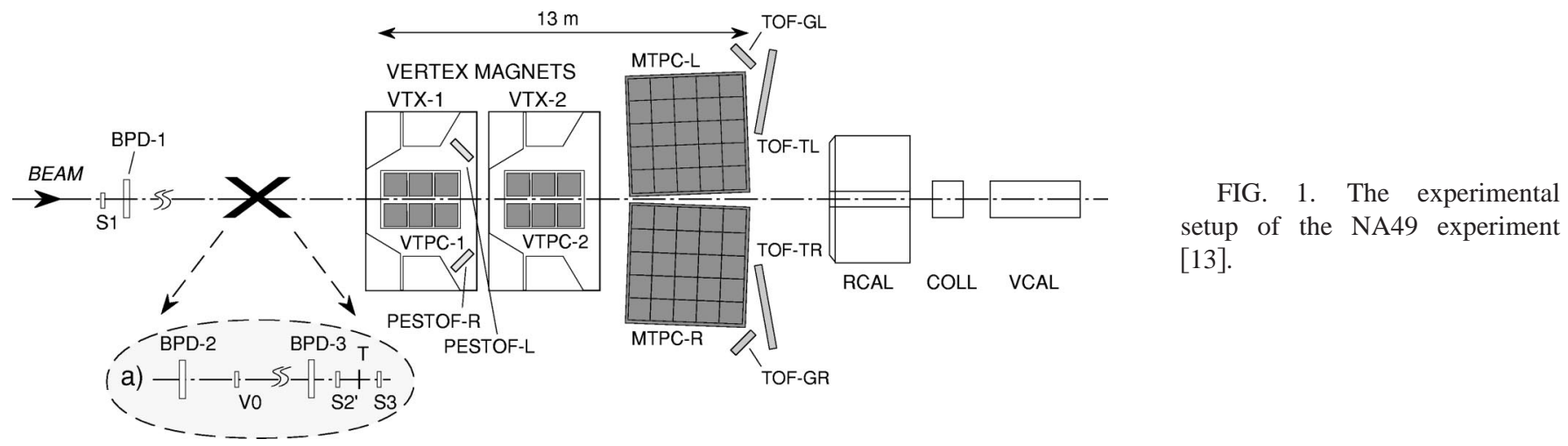

sions at top SPS $(158 \mathrm{~A} \mathrm{GeV})$ and AGS $(11 A \mathrm{GeV})$ energies have suggested [4] that anomalies in pion and strangeness production may be located between these energies. The study of this hypothesis is the motivation for a dedicated energy scan at the CERN SPS [9]. Within this ongoing project NA49 has recorded central $\mathrm{Pb}+\mathrm{Pb}$ collisions at 40 and 80 $A \mathrm{GeV}$ during the heavy ion runs in 1999 and 2000, respectively. The data at the top SPS energy $(158 \mathrm{~A} \mathrm{GeV})$ were taken in previous SPS runs. In this paper we report final results on the energy dependence of charged pion and kaon production. A preliminary analysis was presented in Refs. $[10,11]$. Pseudorapidity spectra of charged particles produced in $\mathrm{Pb}+\mathrm{Pb}$ collisions at 40 and $158 \mathrm{~A} \mathrm{GeV}$ were recently published by the NA50 experiment [12].

The energy scan program at the CERN SPS will be completed in 2002 by taking data at 20 and $30 \mathrm{~A} \mathrm{GeV}$.

\section{EXPERIMENTAL SETUP}

The NA49 experimental setup [13] is shown in Fig. 1. It consists of four large volume time projection chambers (TPCs). Two of these, the vertex TPCs (VTPC-1 and VTPC2 ), are placed in the magnetic field of two superconducting dipole magnets. This allows separation of positively and negatively charged tracks and a precise measurement of the particle momenta $p$ with a resolution of $\sigma(p) / p^{2} \cong(0.3-7)$ $\times 10^{-4}(\mathrm{GeV} / c)^{-1}$. The other two TPCs (MTPC-L and MTPC-R), positioned downstream of the magnets were optimized for high precision measurement of the ionization energy loss $d E / d x$ (relative resolution of about $4 \%$ ), which provides a means of determining the particle mass. The particle identification capability of the MTPCs is augmented by two time of flight (TOF) detector arrays with a resolution $\sigma_{\mathrm{TOF}} \cong 60$ ps.

The acceptance of the NA49 detector is illustrated in Fig. 2 , where we show the distribution of reconstructed unidentified particles versus the total momentum $p$ and transverse momentum $p_{T}$ for the $80 \mathrm{~A} \mathrm{GeV}$ data. The light (dark) shaded region shows the coverage of the MTPCs (TOF) detectors. The resolution of the $d E / d x$ measurement allows hadron identification in the MTPCs for $p>4 \mathrm{GeV}$. At each incident energy the TOF acceptance for kaons was kept at midrapidity by lowering the nominal magnetic field $[B(\mathrm{VTX}-1,2) \approx(1.5$, 1.1) $\mathrm{T}$ at $158 \mathrm{~A} \mathrm{GeV}]$ in proportion to the beam energy. Data were taken for both field polarities.
The target, a thin lead foil $\left(224 \mathrm{mg} / \mathrm{cm}^{2} \cong 1 \%\right.$ of the interaction length), was positioned about $80 \mathrm{~cm}$ upstream from VTPC-1.

Central collisions were selected by a trigger using information from a downstream calorimeter (VCAL), which measured the energy of the projectile spectator nucleons. The geometrical acceptance of the VCAL calorimeter was adjusted for each energy in order to cover the projectile spectator region by a proper setting of a collimator (COLL) $[13,14]$.

\section{ANALYSIS}

Raw $K^{+}$and $K^{-}$yields were extracted from fits of the distributions of $d E / d x$ and TOF (where available) in narrow bins of momentum and transverse momentum. The spectra at midrapidity are obtained using the combined $d E / d x$ and $\mathrm{TOF}$ information ( $\mathrm{TOF}+d E / d x$ analysis). The resulting distributions were corrected for geometrical acceptance, losses due to in-flight decays, and reconstruction efficiency. The first two corrections are calculated using the detector simu-

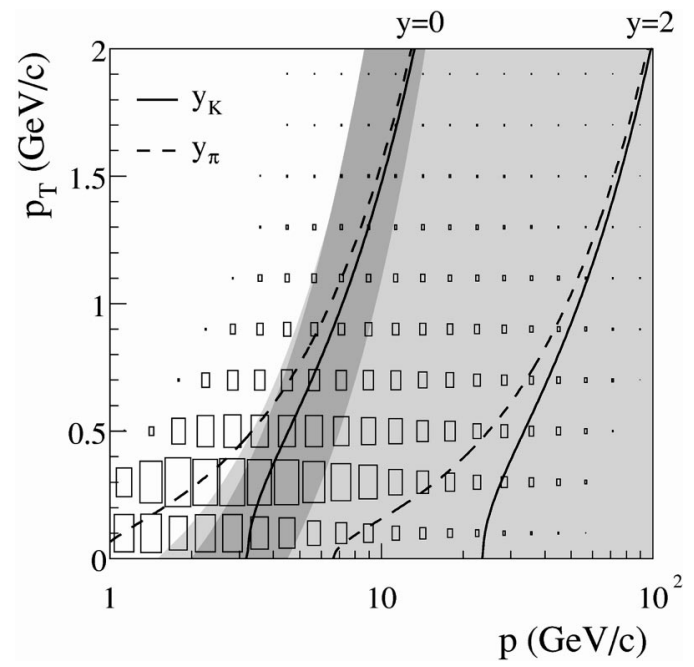

FIG. 2. Distribution of accepted unidentified particles versus the total momentum $p$ and the transverse momentum $p_{T}$ at $80 A \mathrm{GeV}$ beam energy. The light (dark) shaded area indicates the acceptance of the MTPCs (TOF detectors). Also shown are two isolines of constant rapidity $y$ for kaons (full curves) and pions (dashed curves). 
lation package GEANT [15]. The procedure used for the efficiency calculation is discussed below, in the context of the pion analysis.

For pions at midrapidity the acceptance of the $d E / d x$ and $\mathrm{TOF}+d E / d x$ methods is limited to the high $p_{T}$ region (see Fig. 2). To obtain the $\pi^{-}$spectra, yields of all negatively charged particles were determined as a function of rapidity (calculated assuming the $\pi$ mass) and $p_{T}$. The contamination of $K^{-}, \bar{p}$, and $e^{-}$from the interaction vertex as well as nonvertex hadrons originating from strange particle decays and secondary interactions was subtracted using two different methods.

In the first method each track measured in the TPCs was extrapolated back to the target plane. The distance between the track and the interaction vertex was calculated in this plane (track impact parameter). The impact parameter distributions were used to establish cuts for the selection of tracks from the interaction vertex and to estimate the contribution of the remaining nonvertex tracks. A correction for $K^{-}$contamination was calculated using parametrized $K^{-}$spectra.

In the second method all tracks fitted to the interaction vertex were accepted. The necessary corrections were calculated based on a Venus [16] simulation of central $\mathrm{Pb}+\mathrm{Pb}$ collisions. The Venus events were processed by GEANT [15] and the NA49 software which simulates the TPC response and produces files in raw data format. These events were reconstructed and the reconstructed tracks were matched to the Venus input. The obtained correction was scaled by a factor which matches the simulated Venus and the measured hadron yields. The corrections determined using both methods are compatible. The Venus based correction, amounting to $20-25 \%$, is used for the data presented in this paper.

The $\pi^{+}$spectra were not analyzed, because the positive particles have a large and uncertain contribution of protons.

The resulting $K^{ \pm}$and $\pi^{-}$spectra were corrected for geometrical acceptance, and losses due to inefficiencies of the tracking algorithms and quality cuts. These losses were determined using the "embedding" method. Events containing a few tracks were generated and processed by the simulation software. The resulting raw data were embedded into real events. The combined raw data were reconstructed and the input tracks were matched with the reconstructed ones. The calculated losses are about 5\%.

In order to reduce the systematic errors, the analysis has been restricted to regions of phase space where the background and efficiency corrections are small and approximately uniform. The systematic errors were estimated to be below 10\%. This estimate is based on the comparison of results obtained using different detectors (TOF, TPCs), and varying cuts and correction strategies (see above). Additionally, data taken at the two magnetic field polarities were analyzed and the results were found to agree within 5\%. Note that the same experimental procedure was used to obtain results at all three energies. Thus to a large extent the systematic uncertainties are common for the NA49 measurements.

The average number of wounded nucleons $[17]\left\langle N_{W}\right\rangle$ (the notation $\langle\cdots\rangle$ will be used to denote the mean multiplicity in full phase space throughout the paper) as given in Table I
TABLE I. Numbers of analyzed events, cross sections of selected central interactions as percentage of total inelastic cross section $\left(\sigma^{I N E L}=7.15 \mathrm{~b}\right)$, and mean numbers of wounded nucleons for the selected central $\mathrm{Pb}+\mathrm{Pb}$ collisions at 40,80 , and $158 \mathrm{~A} \mathrm{GeV}$. The first error given in the last column is statistical, the second systematic.

\begin{tabular}{cccc}
\hline \hline $\begin{array}{c}\text { Energy } \\
(A \mathrm{GeV})\end{array}$ & $\begin{array}{c}\text { Number of } \\
\text { events }\end{array}$ & $\begin{array}{c}\sigma^{C E N T} / \sigma^{\text {INEL }} \\
(\%)\end{array}$ & $\left\langle N_{W}\right\rangle$ \\
\hline 40 & $4 \times 10^{5}$ & 7.2 & $349 \pm 1 \pm 5$ \\
80 & $3 \times 10^{5}$ & 7.2 & $349 \pm 1 \pm 5$ \\
158 & $4 \times 10^{5}$ & 5.0 & $362 \pm 1 \pm 5$ \\
\hline \hline
\end{tabular}

was not directly measured but was calculated using the Fritiof model [18]. In an unbiased sample of generated inelastic interactions a subsample of central events was selected by applying a cut in the number of projectile spectators. This cut was adjusted such that the selected fraction was equal to the fraction of all inelastic interactions accepted by the central trigger in the experiment. The value of $\left\langle N_{W}\right\rangle$ was then cal-

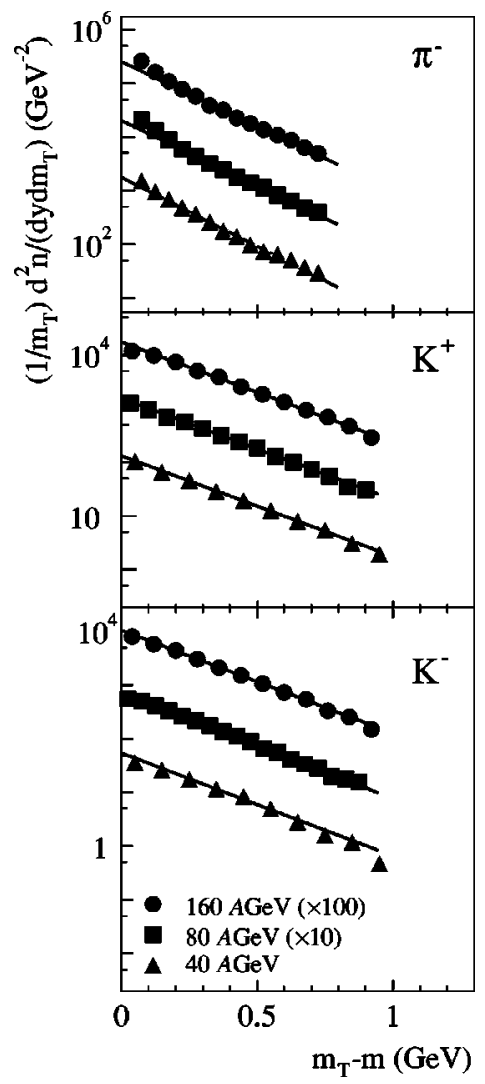

FIG. 3. Transverse mass spectra of $\pi^{-}, K^{+}$, and $K^{-}$mesons produced at midrapidity $(|y|<0.1$ for kaons in the TOF $+d E / d x$ analysis, and $0<y<0.2$ for pions) in central $\mathrm{Pb}+\mathrm{Pb}$ collisions at 40 (triangles), 80 (squares), and 158 (circles) $A \mathrm{GeV}$. The values for $80 A \mathrm{GeV}$ and $158 A \mathrm{GeV}$ are scaled by factors of 10 and 100 , respectively. The lines are exponential fits to the spectra [see text, Eq. (1)] in the interval $0.2 \mathrm{GeV}<m_{T}-m<0.7 \mathrm{GeV}$. Statistical errors are smaller than the symbol size. The systematic errors are $\pm 5 \%$ in the region used for the fit and reach $\pm 10 \%$ at the edges of the acceptance. 
TABLE II. Inverse slope parameters $T$ of the transverse mass spectra fitted in the interval $0.2 \mathrm{GeV}<m_{T}-m<0.7 \mathrm{GeV}$ at midrapidity $(|y|<0.1$ for kaons in the TOF $+d E / d x$ analysis, and $0<y$ $<0.2$ for pions), rapidity densities $d n / d y$ averaged over the interval $|y|<0.6$ as well as total mean multiplicities of $\pi^{-}, \pi^{+}, K^{-}$and $K^{+}$mesons produced in central $\mathrm{Pb}+\mathrm{Pb}$ collisions at 40,80 , and 158 $A \mathrm{GeV}$. The first error is statistical, the second systematic. Note that $\left\langle\pi^{+}\right\rangle$is not directly measured.

\begin{tabular}{lccc}
\hline \hline & $40 A \mathrm{GeV}$ & $80 A \mathrm{GeV}$ & $158 A \mathrm{GeV}$ \\
\hline$T\left(\pi^{-}\right)(\mathrm{MeV})$ & $169 \pm 2 \pm 10$ & $179 \pm 3 \pm 10$ & $180 \pm 3 \pm 10$ \\
$T\left(K^{+}\right)(\mathrm{MeV})$ & $232 \pm 3 \pm 6$ & $230 \pm 5 \pm 6$ & $232 \pm 4 \pm 6$ \\
$T\left(K^{-}\right)(\mathrm{MeV})$ & $226 \pm 3 \pm 6$ & $217 \pm 3 \pm 6$ & $226 \pm 9 \pm 6$ \\
$d n / d y\left(\pi^{-}\right)$ & $106.1 \pm 0.4 \pm 6$ & $140.4 \pm 0.5 \pm 7$ & $175.4 \pm 0.7 \pm 9$ \\
$d n / d y\left(\pi^{+}\right)$ & $96.6 \pm 0.4 \pm 6$ & $132.0 \pm 0.5 \pm 7$ & $170.1 \pm 0.7 \pm 9$ \\
$d n / d y\left(K^{+}\right)$ & $20.1 \pm 0.3 \pm 1.0$ & $24.6 \pm 0.2 \pm 1.2$ & $29.6 \pm 0.3 \pm 1.5$ \\
$d n / d y\left(K^{-}\right)$ & $7.58 \pm 0.12 \pm 0.4$ & $11.7 \pm 0.10 \pm 0.6$ & $16.8 \pm 0.2 \pm 0.8$ \\
$\left\langle\pi^{-}\right\rangle$ & $322 \pm 3 \pm 16$ & $474 \pm 5 \pm 23$ & $639 \pm 17 \pm 31$ \\
$\left\langle\pi^{+}\right\rangle$ & $293 \pm 3 \pm 15$ & $446 \pm 5 \pm 22$ & $619 \pm 17 \pm 31$ \\
$\left\langle K^{+}\right\rangle$ & $59.1 \pm 1.9 \pm 3$ & $76.9 \pm 2 \pm 4$ & $103.0 \pm 5 \pm 5$ \\
$\left\langle K^{-}\right\rangle$ & $19.2 \pm 0.5 \pm 1.0$ & $32.4 \pm 0.6 \pm 1.6$ & $51.9 \pm 1.9 \pm 3$ \\
\hline \hline
\end{tabular}

culated for the Fritiof $\mathrm{Pb}+\mathrm{Pb}$ collisions selected in this way. Finally it was verified that the $\left\langle N_{W}\right\rangle$ value for central $\mathrm{Pb}+\mathrm{Pb}$ collisions at $158 \mathrm{~A} \mathrm{GeV}$ agrees (within several percent) with the total number of net baryons determined from the participant domain (in the rapidity interval $-2.6<y<2.6$ ) for these collisions [19].

Note that in this paper $y$ denotes the rapidity of a particle in the collision center-of-mass system (c.m.s.).

Table I summarizes the parameters characterizing the data samples used in this analysis.

\section{RESULTS}

Spectra of transverse mass $m_{T}=\sqrt{p_{T}^{2}+m^{2}}$ ( $m$ is the rest mass of the particle) for $K^{+}, K^{-}$(TOF $+d E / d x$ analysis) and $\pi^{-}$mesons produced near midrapidity $(|y|<0.1$ for kaons and $0<y<0.2$ for pions) in central $\mathrm{Pb}+\mathrm{Pb}$ collisions at 40, 80, and $158 \mathrm{~A} \mathrm{GeV}$ are shown in Fig. 3. The full lines indicate a fit of the function

$$
\frac{d n}{m_{T} d m_{T} d y}=C \exp \left(-\frac{m_{T}}{T}\right)
$$

to the data in the range $0.2 \mathrm{GeV}<m_{T}-m<0.7 \mathrm{GeV}$. The values obtained for the inverse slope parameter $T$ are presented in Table II. The $T$ parameter is smaller for pions than for kaons. A weak increase of $T$ with increasing energy is suggested by the pion data, whereas no significant change is seen for kaons.

The rapidity distributions $d n / d y$ plotted in Fig. 4 were obtained by summing the measured $m_{T}$ spectra and using the fitted exponential function [Eq. (1)] to extrapolate to full $m_{T}$. For most bins the necessary correction is small $(\cong 5 \%)$. The values of $d n / d y$ at midrapidity $(|y|<0.6)$ are given in Table II. An increase of rapidity density with energy is seen for all particles. The rapidity spectra were param-

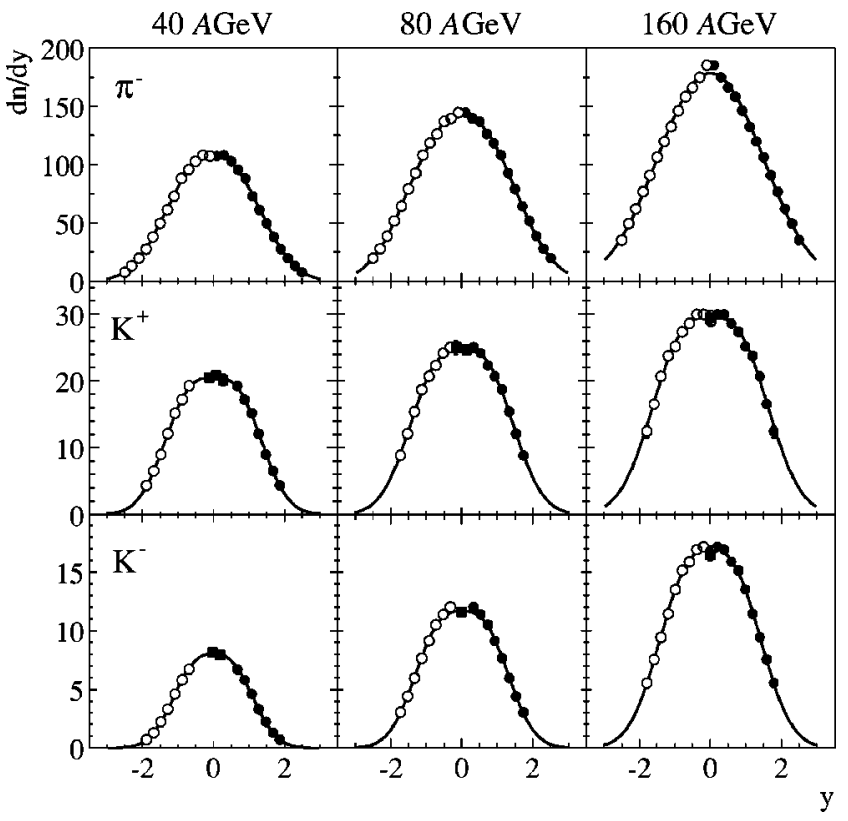

FIG. 4. Rapidity distributions of $\pi^{-}, K^{+}$, and $K^{-}$mesons produced in central $\mathrm{Pb}+\mathrm{Pb}$ collisions at 40,80 , and $158 A \mathrm{GeV}$. For kaons, squares, and circles indicate the results of TOF $+d E / d x$ and $d E / d x$ only analyses, respectively. The closed symbols indicate measured points, open points are reflected with respect to midrapidity. The lines indicate $2-\mathrm{G}$ fits to the spectra [see Eq. (2)]. The plotted errors, which are mostly smaller than the symbol size, are statistical only, the systematic errors are $\pm 5 \%$.

etrized by the sum of two Gauss distributions placed symmetrically with respect to midrapidity:

$$
\frac{d n}{d y}=N\left[\exp \left(-\frac{\left(y-y_{0}\right)^{2}}{2 \sigma^{2}}\right)+\exp \left(-\frac{\left(y+y_{0}\right)^{2}}{2 \sigma^{2}}\right)\right] .
$$

The results of the fits are indicated by the full lines in Fig. 4 and the obtained values of the parameters $N, y_{0}$, and $\sigma$ are given in Table III. Since both $y_{0}$ and $\sigma$ increase with increasing energy, the width of the observed rapidity distributions clearly increases with energy. The mean multiplicities in full phase space were calculated by integrating the parametrized

TABLE III. Fitted parameters of the 2-G parametrization [see text, Eq. (2)] of rapidity distributions measured for $\pi^{-}, K^{-}$, and $K^{+}$mesons produced in central $\mathrm{Pb}+\mathrm{Pb}$ collisions at 40,80 , and 158 $A \mathrm{GeV}$. Only statistical errors are given.

\begin{tabular}{lccc}
\hline \hline & $40 A \mathrm{GeV}$ & $80 A \mathrm{GeV}$ & $158 A \mathrm{GeV}$ \\
\hline$N\left(\pi^{-}\right)$ & $74.0 \pm 0.5$ & $97.0 \pm 0.7$ & $107.6 \pm 1.8$ \\
$N\left(K^{+}\right)$ & $16.2 \pm 0.4$ & $19.3 \pm 0.3$ & $23.4 \pm 0.6$ \\
$N\left(K^{-}\right)$ & $6.03 \pm 0.13$ & $9.16 \pm 0.12$ & $12.8 \pm 0.3$ \\
$\sigma\left(\pi^{-}\right)$ & $0.872 \pm 0.005$ & $0.974 \pm 0.007$ & $1.18 \pm 0.02$ \\
$\sigma\left(K^{+}\right)$ & $0.725 \pm 0.016$ & $0.792 \pm 0.018$ & $0.88 \pm 0.04$ \\
$\sigma\left(K^{-}\right)$ & $0.635 \pm 0.011$ & $0.705 \pm 0.010$ & $0.81 \pm 0.02$ \\
$y_{0}\left(\pi^{-}\right)$ & $0.666 \pm 0.006$ & $0.756 \pm 0.006$ & $0.72 \pm 0.02$ \\
$y_{0}\left(K^{+}\right)$ & $0.694 \pm 0.008$ & $0.742 \pm 0.008$ & $0.839 \pm 0.012$ \\
$y_{0}\left(K^{-}\right)$ & $0.569 \pm 0.010$ & $0.668 \pm 0.005$ & $0.727 \pm 0.010$ \\
\hline \hline
\end{tabular}




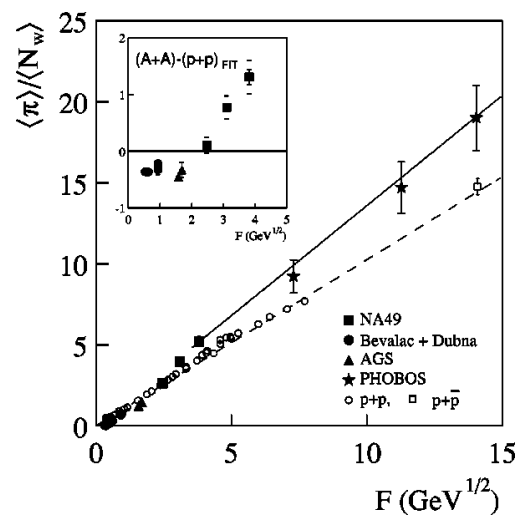

FIG. 5. Dependence of the total pion multiplicity per wounded nucleon on Fermi's energy measure $F$ (see text) for central $A+A$ collisions (closed symbols) and inelastic $p+p(\bar{p})$ interactions (open symbols). The results of NA49 are indicated by squares. The full line shows a linear fit through the origin to the $A+A$ data at and above $158 \mathrm{~A} \mathrm{GeV}$. The inset shows the difference between the results for $A+A$ collisions and the straight line parametrization of $p$ $+p(\bar{p})$ data (dashed line). The inner error bars on the NA49 points indicate the statistical uncertainty and the outer error bars the statistical and systematic uncertainty added in quadrature.

rapidity spectra. The resulting numbers are given in Table II. The mean multiplicity of $\pi^{+}$mesons given in Table II was calculated by scaling $\left\langle\pi^{-}\right\rangle$by the $\pi^{+} / \pi^{-}$ratio $(0.91,0.94$, and 0.97 at 40,80 , and $158 \mathrm{~A} \mathrm{GeV}$, respectively) measured in the region where both $d E / d x$ and TOF measurements are available. Similar ratios (within $2 \%$ ) are predicted by the Venus model [16]. We have also checked, with the Venus model, that the $\pi^{+} / \pi^{-}$ratio of total multiplicities is, within $1.5 \%$, equal to the ratio in the TOF $+d E / d x$ acceptance. Results on $d n / d y$ and inverse slope parameters near midrapidity at $158 \mathrm{~A} \mathrm{GeV}$ are compatible with previously published measurements [20].

\section{ENERGY DEPENDENCE}

The energy dependence of the mean pion multiplicity $\langle\pi\rangle=1.5\left(\left\langle\pi^{+}\right\rangle+\left\langle\pi^{-}\right\rangle\right)$is shown in Fig. 5. In this figure the ratio $\langle\pi\rangle /\left\langle N_{W}\right\rangle$ is plotted as a function of the collision energy, expressed by Fermi's measure [21]: $F \equiv\left(\sqrt{s_{N N}}\right.$ $\left.-2 m_{N}\right)^{3 / 4} / \sqrt{s_{N N}^{1 / 4}}$, where $\sqrt{s_{N N}}$ is the c.m.s. energy per nucleon-nucleon pair and $m_{N}$ the rest mass of the nucleon. Measurements by NA49 are compared to results from other experiments on central nucleus-nucleus collisions [4,22,23] and to a compilation of data from $p+p(\bar{p})$ interactions (see references in Ref. [4]).

One observes that the mean pion multiplicity in $p$ $+p(\bar{p})$ interactions is approximately proportional to $F$; the dashed line in Fig. 5 indicates a fit of the form $\langle\pi\rangle /\left\langle N_{W}\right\rangle$ $=a F$ to the data, yielding $a=1.025 \pm 0.005 \mathrm{GeV}^{-1 / 2}$. For central $A+A$ collisions the dependence is more complicated and it can not be fitted by a single linear function $\left(\chi^{2} / \mathrm{DOF}\right.$ $\approx 18$ ). Below $40 A \mathrm{GeV}$ the ratio $\langle\pi\rangle /\left\langle N_{W}\right\rangle$ in $A+A$ collisions is lower than in $p+p$ interactions (pion suppression), while at higher energies $\langle\pi\rangle /\left\langle N_{W}\right\rangle$ is larger in $A+A$ colli-

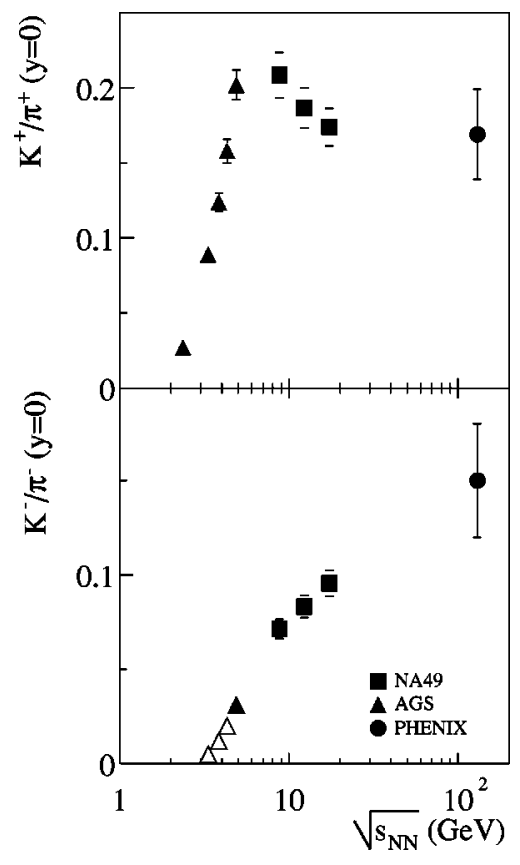

FIG. 6. Energy dependence of the midrapidity $K^{+} / \pi^{+}$and $K^{-} / \pi^{-}$ratios in central $\mathrm{Pb}+\mathrm{Pb}$ and $\mathrm{Au}+\mathrm{Au}$ collisions. The results of NA49 are indicated by squares. Open triangles indicate the $A$ $+A$ results for which preliminary data were used [25]. The errors on the NA49 points are the statistical and systematic errors added in quadrature. The statistical errors are smaller than the symbol size.

sions than in $p+p(\bar{p})$ interactions (pion enhancement). In the region between AGS and the lowest SPS energy (15-40 $A \mathrm{GeV})$ the slope changes from $a=1.01$ $\pm 0.04 \mathrm{GeV}^{-1 / 2}\left(\chi^{2} / \mathrm{DOF} \approx 0.9\right)$ for the fit to the points up to the top AGS energy to $a=1.36 \pm 0.03 \mathrm{GeV}^{-1 / 2}\left(\chi^{2} / \mathrm{DOF}\right.$ $\approx 0.2$ ) for the fit to the top SPS energy and the RHIC data points [23]. The fit to the top SPS and RHIC points is indicated by the full line in Fig. 5. The transition from pion suppression to pion enhancement is demonstrated more clearly in the inset of Fig. 5, where the difference between $\langle\pi\rangle /\left\langle N_{W}\right\rangle$ for $A+A$ collisions and the straight line parametrization of the $p+p$ data is plotted as a function of $F$ up to the highest SPS energy.

Midrapidity and full phase space kaon to pion ratios are shown as a function of $\sqrt{s_{N N}}$ in Figs. 6 and 7 [4,22,24], respectively. A monotonic increase with $\sqrt{s_{N N}}$ of the $K^{-} / \pi^{-}$ ratio is measured. For the $K^{+} / \pi^{+}$ratio, a very different behavior is observed: a steep increase in the low (AGS [22]) energy region is followed by a maximum around $40 \mathrm{~A} \mathrm{GeV}$. The measurement at RHIC indicates that the $K^{+} / \pi^{+}$ratio stays nearly constant starting from the top SPS energy. For comparison, the results on the $\left\langle K^{+}\right\rangle /\left\langle\pi^{+}\right\rangle$ratio in $p+p$ interactions [4] are also shown in Fig. 7. The $p+p$ data have rather large experimental uncertainties [4], but suggest a monotonic increase of the ratio. It should be noted that the $\left\langle K^{+}\right\rangle /\left\langle\pi^{+}\right\rangle$ratio is expected to be similar (within about $10 \%$ ) for $p+p, n+p$, and $n+n$ interactions [27].

The energy dependence of the $K^{-} / K^{+}$ratio at midrapidity is shown in Fig. 8. The ratio increases with $\sqrt{s_{N N}}$ from about 0.15 at low AGS energy [22] to about 0.5 at SPS energies and reaches about 0.9 at $\mathrm{RHIC}$ [24]. 


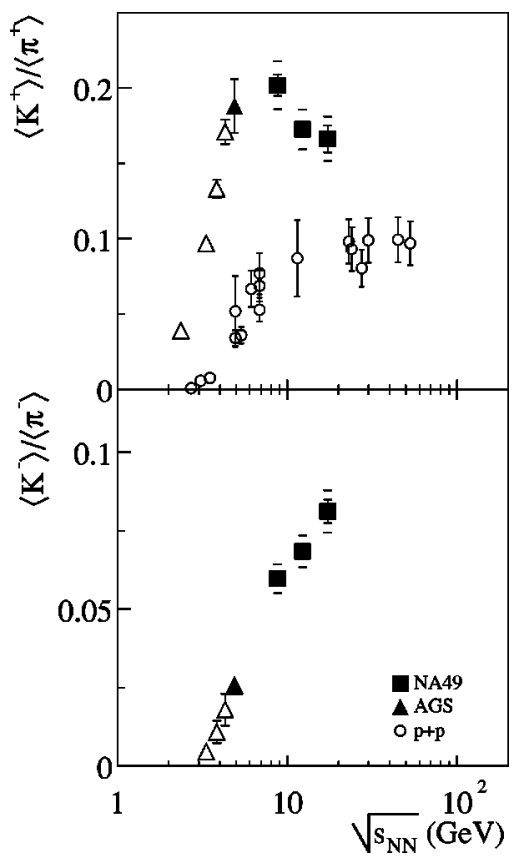

FIG. 7. Energy dependence of full phase space $\left\langle K^{+}\right\rangle /\left\langle\pi^{+}\right\rangle$and $\left\langle K^{-}\right\rangle /\left\langle\pi^{-}\right\rangle$ratios in central $\mathrm{Pb}+\mathrm{Pb}(\mathrm{Au}+\mathrm{Au})$ collisions. The results of NA49 are indicated by squares. The data for $p+p$ interactions are shown by open circles for comparison. Open triangles indicate the $A+A$ results for which a substantial extrapolation was necessary [26]. The inner error bars on the NA49 points indicate the statistical uncertainty and the outer error bars the statistical and systematic uncertainty added in quadrature.

The difference between the dependence of the $K^{+}$and $K^{-}$ yields on $\sqrt{s_{N N}}$ can be attributed to their different sensitivity to the baryon density. Kaons $\left(K^{+}\right.$and $\left.K^{0}\right)$ carry a dominant fraction of all produced $\bar{s}$ quarks (more than $95 \%$ in $\mathrm{Pb}+\mathrm{Pb}$ collisions at $158 \mathrm{~A} \mathrm{GeV}$ if open strangeness is considered). Therefore the $K^{+}$yield $\left(\left\langle K^{+}\right\rangle \cong\left\langle K^{0}\right\rangle\right.$ in approximately isospin symmetric collisions of heavy nuclei) is nearly proportional to the total strangeness production and only weakly sensitive to the baryon density. As a significant fraction of $s$

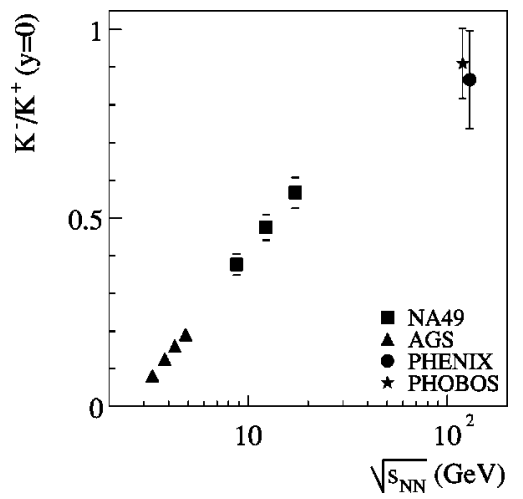

FIG. 8. Energy dependence of the midrapidity $K^{-} / K^{+}$ratio in central $\mathrm{Pb}+\mathrm{Pb}(\mathrm{Au}+\mathrm{Au})$ collisions. The results of NA49 are indicated by squares. The errors on the NA49 points are the statistical and systematic errors added in quadrature. The statistical errors are smaller than the symbol size.

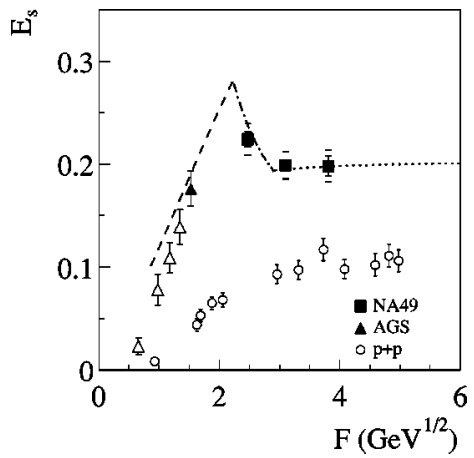

FIG. 9. Energy dependence of the $E_{S}=(\langle\Lambda\rangle+\langle K+\bar{K}\rangle) /\langle\pi\rangle$ ratio in central $\mathrm{Pb}+\mathrm{Pb}(\mathrm{Au}+\mathrm{Au})$ collisions and $p+p$ interactions. The results of NA49 are indicated by squares. Open triangles indicate the $A+A$ results for which a substantial extrapolation was necessary [26]. The experimental results on $A+A$ collisions are compared with the predictions of the statistical model of the early stage (line) [6]. Different line styles indicate predictions in the energy domains in which confined matter (dashed line), mixed phase (dash-dotted line), and QGP (dotted line) are created at the early stage of the collisions. The inner error bars on the NA49 points indicate the statistical uncertainty and the outer error bars the statistical and systematic uncertainty added in quadrature.

quarks (e.g., $\approx 50 \%$ in central $\mathrm{Pb}+\mathrm{Pb}$ collisions at $158 \mathrm{~A} \mathrm{GeV}$ ) is carried by hyperons, the number of produced antikaons $\left(K^{-}\right.$and $\left.\bar{K}^{0}\right)$ is sensitive to both the strangeness yield and the baryon density.

In Fig. 9 an alternative measure of the strangeness to pion ratio, $E_{S}=(\langle\Lambda\rangle+\langle K+\bar{K}\rangle) /\langle\pi\rangle$, is plotted as a function of $F$ for $A+A$ collisions [22] and $p+p$ interactions [4]. For $A$ $+A$ collisions the $\Lambda$ multiplicity, when not published (e.g., for the NA49 points), was estimated as $\langle\Lambda\rangle=\left(\left\langle K^{+}\right\rangle\right.$ $\left.-\left\langle K^{-}\right\rangle\right) / 0.8$, based on strangeness conservation and approximate isospin symmetry of the colliding nuclei [27]. The wealth of data on $\Lambda$ and $K_{S}^{0}\left(K_{S}^{0}=0.5\left\langle K^{0}+\bar{K}^{0}\right\rangle \approx 0.5\left\langle K^{+}\right.\right.$ $\left.+K^{-}\right\rangle$) production in $p+p$ interactions [4] allows a much more precise determination of $E_{S}$ (open circles in Fig. 9) than of the $\left\langle K^{+}\right\rangle /\left\langle\pi^{+}\right\rangle$ratio (open circles in Fig. 7). By construction, $E_{S}$ should be almost independent (an expected variation of several percent) of the charge composition of colliding nuclei. One may conclude from Figs. 7 and 9 that a nonmonotonic energy dependence (or a sharp turnover) of the total strangeness to pion ratio appears to be a special property of heavy ion collisions, which is not observed in elementary interactions.

\section{COMPARISON WITH MODELS}

The energy dependence of pion and strangeness yields was discussed within various approaches to nucleus-nucleus collisions. In this section we compare our results with published model predictions.

It was suggested $[4,5]$ that a transition to a deconfined state of matter may cause anomalies in the energy dependence of pion and strangeness production. This led to the formulation of the statistical model of the early stage (SMES) [5,6], which is based on the assumption that the 
system created at the early stage (be it confined matter or a QGP) is in equilibrium and a transition from a reaction with purely confined matter to a reaction with a QGP at the early stage occurs when the transition temperature $T_{C}$ is reached. For $T_{C}$ values of $170-200 \mathrm{MeV}$ the transition region ranges between $15-60 A \mathrm{GeV}[6]$.

Due to the assumed generalized Fermi-Landau initial conditions $[6,21,28]$ the $\langle\pi\rangle /\left\langle N_{W}\right\rangle$ ratio (a measure of entropy per baryon) increases approximately linearly with $F$ outside the transition region. The slope parameter is proportional to $g^{1 / 4}$ [5], where $g$ is an effective number of internal degrees of freedom at the early stage. In the transition region a steepening of the pion energy dependence is expected, because of activation of a large number of partonic degrees of freedom. This is, in fact, observed in the data on central $\mathrm{Pb}+\mathrm{Pb}(\mathrm{Au}$ $+\mathrm{Au})$ collisions, where the steepening starts in the range 15-40 A GeV (see Fig. 5). The linear dependence on $F$ is obeyed by the data at lower and higher energies (including RHIC). An increase of the slope by a factor of about 1.3 is measured (see Sec. V), which corresponds to an increase of the effective number of internal degrees of freedom by a factor of $1.3^{4} \cong 3$, within the SMES [5].

In the SMES model the $\left\langle K^{+}\right\rangle /\left\langle\pi^{+}\right\rangle$and $E_{S}$ ratios are roughly proportional to the total strangeness to entropy ratio, which is assumed to be preserved from the early stage till freeze-out. At low collision energies the strangeness to entropy ratio steeply increases with collision energy, due to the low temperature at the early stage $\left(T<T_{C}\right)$ and the high mass of the carriers of strangeness $\left(m_{S} \cong 500 \mathrm{MeV}\right.$, the kaon mass) in the confined state. When the transition to a QGP is crossed $\left(T>T_{C}\right)$, the mass of the strangeness carriers is significantly reduced ( $m_{S} \cong 170 \mathrm{MeV}$, the strange quark mass). Due to the low mass $\left(m_{S}<T\right)$ the strangeness yield becomes (approximately) proportional to the entropy, and the strangeness to entropy (or pion) ratio is independent of energy. This leads to a "jump" in the energy dependence from the larger value for confined matter at $T_{C}$ to the QGP value. Thus, within the SMES, the measured nonmonotonic energy dependence of the strangeness to entropy ratio is followed by a saturation at the QGP value (see Figs. 7 and 9), which is a direct consequence of the onset of deconfinement taking place at about $40 \mathrm{~A} \mathrm{GeV}$.

Numerous models have been developed to explain hadron production in reactions of heavy nuclei without explicitly invoking a transient QGP phase. The simplest one is the statistical hadron gas model [29] where independent of the collision energy the hadrochemical freeze-out creates a hadron gas in equilibrium [30]. The temperature, baryon chemical potential and hadronization volume are free parameters of the model, which are fitted to the data at each energy. In this formulation, the hadron gas model does not predict the energy dependence of hadron production. Recently, an extension of the model was proposed, in which it is assumed that the values of the thermal parameters (temperature and baryon chemical potential) evolve smoothly with the collision energy [31]. The energy dependence calculated within this extended hadron gas model for the $\left\langle K^{+}\right\rangle /\left\langle\pi^{+}\right\rangle$ratio is compared to the experimental results in Fig. 10. Due to its construction, the prevailing trend in the data is reproduced

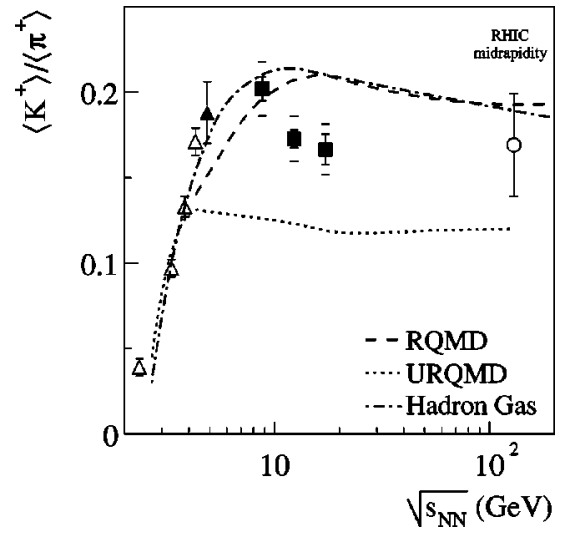

FIG. 10. Energy dependence of the full phase space $\left\langle K^{+}\right\rangle /\left\langle\pi^{+}\right\rangle$ ratio in central $\mathrm{Pb}+\mathrm{Pb}$ and $\mathrm{Au}+\mathrm{Au}$ collisions. The experimental results taken from Fig. 7 are compared to model predictions: RQMD [33] (dashed line), UrQMD [35] (dotted line), and the extended hadron gas model [31] (dash-dotted line).

by the model, but the decrease of the ratio between 40 and $158 \mathrm{~A} \mathrm{GeV}$ is not well described. The measured strangeness to pion yield in central $\mathrm{Pb}+\mathrm{Pb}$ collisions at $158 \mathrm{~A} \mathrm{GeV}$ is about $25 \%$ lower than the expectation for the fully equilibrated hadron gas $[31,32]$.

Several dynamical hadron-string models have been developed to study hadron production in $A+A$ collisions. These models treat the elementary collisions with a string-hadronic framework as a starting point. The models are then extended with effects which are expected to be relevant in $A+A$ collisions (such as string-string interactions and hadronic rescattering). The predictions of the RQMD [33,34] and the UrQMD $[35,36]$ models are shown in Fig. 10. It is seen that RQMD, like the hadron gas model, fails to describe the decrease of the $\left\langle K^{+}\right\rangle /\left\langle\pi^{+}\right\rangle$ratio in the SPS energy range. The UrQMD model predicts a ratio which, above $\sqrt{s_{N N}} \cong 5 \mathrm{GeV}$, does not show any sizable energy dependence and which is significantly lower (e.g., by about $40 \%$ at $40 \mathrm{~A} \mathrm{GeV}$ ) than the data. This is mainly due to the fact that UrQMD overestimates pion production at SPS energies by more than $30 \%$ [36].

The RQMD prediction of the energy dependence of the $\mathrm{K}^{+} / \pi^{+}$ratio at midrapidity is shown in Fig 11 . The model also fails to reproduce the experimental data both in shape and magnitude. In addition, Fig. 11 presents the prediction of the HSD model [37] (another version of the dynamical hadron-string approach) which shows a monotonic increase of the $K^{+} / \pi^{+}$ratio with energy. This trend is very different from the measured one.

\section{SUMMARY}

Results on charged pion and kaon production in central $\mathrm{Pb}+\mathrm{Pb}$ collisions at 40,80 , and $158 \mathrm{AGeV}$ are presented. These are compared with data at lower and higher energies as well as with results from $p+p$ interactions. The mean pion multiplicity per wounded nucleon increases approximately linearly with $s_{N N}^{1 / 4}$ with a change of slope starting in the region 15-40 A GeV. The change from pion suppression 


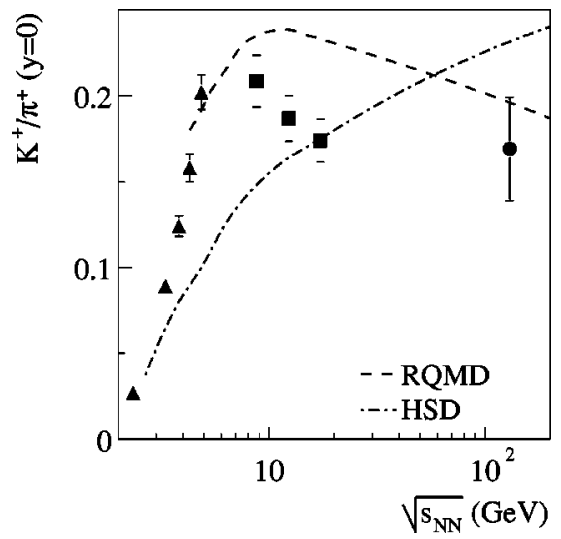

FIG. 11. Energy dependence of the midrapidity $\mathrm{K}^{+} / \pi^{+}$ratio in central $\mathrm{Pb}+\mathrm{Pb}$ and $\mathrm{Au}+\mathrm{Au}$ collisions. The experimental results taken from Fig. 6 are compared with model predictions: RQMD [33] (dashed line) and HSD [37] (dash-dotted line).

with respect to $p+p$ interactions, as observed at low collision energies, to pion enhancement at high energies occurs at about $40 \mathrm{~A} \mathrm{GeV}$. A nonmonotonic energy dependence of the ratio of $K^{+}$to $\pi^{+}$yields is observed, with a maximum close to $40 \mathrm{~A} \mathrm{GeV}$ and an indication of a nearly constant value at higher energies. This characteristic energy dependence is not observed in elementary interactions and seems to be a unique feature of heavy ion collisions. The measured dependences can be related to an increase of the entropy production and a decrease of the strangeness to entropy ratio in central $\mathrm{Pb}+\mathrm{Pb}$ collisions in the low SPS energy range. They can be understood within the statistical model of the early stage of nucleus-nucleus collisions, which assumes that a transient state of deconfined matter is created in $\mathrm{Pb}+\mathrm{Pb}$ collisions for energies larger than about $40 \mathrm{~A} \mathrm{GeV}$. Currently available models without this assumption do not reproduce the measured energy dependence of pion and strangeness production equally well.

\section{ACKNOWLEDGMENTS}

This work was supported by the Director, Office of Energy Research, Division of Nuclear Physics of the Office of High Energy and Nuclear Physics of the U.S. Department of Energy (DE-ACO3-76SFOOO98 and DE-FG0291ER40609), the U.S. National Science Foundation, the Bundesministerium fur Bildung und Forschung, Germany, the Alexander von Humboldt Foundation, the U.K. Engineering and Physical Sciences Research Council, the Polish State Committee for Scientific Research (5 P03B 13820 and 2 P03B 02418), the Hungarian Scientific Research Foundation (T14920 and T23790), the Hungarian National Science Foundation, OTKA (F034707), the EC Marie Curie Foundation, and the Polish-German Foundation.
[1] For review see Quark Matter '99, Proceedings of the 14th International Conference on Ultra-Relativistic NucleusNucleus Collisions, edited by L. Riccati, M. Masera, and E. Vercellin [Nucl. Phys. A661, 1c (1999)].

[2] J. C. Collins and M. J. Perry, Phys. Rev. Lett. 34, 1353 (1975); E. V. Shuryak, Phys. Rep. 61, 71 (1980); 115, 151 (1984).

[3] J. Rafelski and B. Müller, Phys. Rev. Lett. 48, 1066 (1982); T. Matsui and H. Satz, Phys. Lett. B 178, 416 (1986).

[4] M. Gaździcki and D. Röhrich, Z. Phys. C: Part. Fields 65, 215 (1995); 71, 55 (1996), and references therein.

[5] M. Gaździcki, Z. Phys. C: Part. Fields 66, 659 (1995).

[6] M. Gaździcki and M. I. Gorenstein, Acta Phys. Pol. B 30, 2705 (1999), and references therein.

[7] C. M. Hung and E. Shuryak, Phys. Rev. Lett. 75, 4003 (1995).

[8] M. Stephanov, K. Rajagopal, and E. Shuryak, Phys. Rev. D 60, 114028 (1999).

[9] J. Bächler et al., NA49 Collaboration, Report Nos. SPSLC/ P264 and CERN/SPSC 97, 1997.

[10] C. Blume et al., NA49 Collaboration, Nucl. Phys. A698, 104 (2002).

[11] T. Kollegger et al., NA49 Collaboration, J. Phys. G 28, 1689 (2002).

[12] M. C. Abreu et al., NA50 Collaboration, Phys. Lett. B 530, 33 (2002); 530, 43 (2002).

[13] S. Afanasiev et al., NA49 Collaboration, Nucl. Instrum. Methods Phys. Res. A 430, 210 (1999).

[14] H. Appelshauser et al., NA49 Collaboration, Eur. Phys. J. A 2, 383 (1998).

[15] GEANT, Detector Description and Simulation Tool, CERN Program Library Long Writeup W5013.
[16] K. Werner, Phys. Rep. 232, 87 (1993).

[17] A. Bialas, M. Bleszyński, and W. Czyż, Nucl. Phys. B111, 461 (1976).

[18] B. Andersson, G. Gustafson, and Hong Pi, Z. Phys. C: Part. Fields 57, 485 (1993).

[19] H. Appelshauser et al., NA49 Collaboration, Phys. Rev. Lett. 82, 2471 (1999).

[20] I. Bearden et al., NA44 Collaboration, Phys. Lett. B 471, 6 (1999).

[21] E. Fermi, Prog. Theor. Phys. 5, 570 (1950).

[22] L. Ahle et al., E802 Collaboration, Phys. Rev. C 57, 466 (1998); 58, 3523 (1998); 60, 044904 (1999); E866 and E917 Collaboration, Phys. Lett. B 476, 1 (2000); 490, 53 (2000); J. Barrette et al., E877 Collaboration, Phys. Rev. C 62, 024901 (2000); D. Pelte et al., FOPI Collaboration, Z. Phys. A 357, 215 (1997).

[23] B. B. Back et al., PHOBOS Collaboration, Phys. Rev. Lett. 85, 3100 (2000); 87, 102303 (2001); 88, 022302 (2002), the mean charged particle multiplicity in central $\mathrm{Au}+\mathrm{Au}$ collisions at $\sqrt{s}_{N N}=200 \mathrm{GeV}$ was obtained by integrating the published pseudorapidity distribution. At $\sqrt{s}_{N N}=56 \mathrm{GeV}$ the measurement at midrapidity was extrapolated to $4 \pi$ assuming scaling of the shape of the distribution with the beam rapidity. The charged hadron multiplicities were corrected for contributions of charged kaons and protons.

[24] K. Adcox et al., PHENIX Collaboration, Phys. Rev. Lett. 88, 242301 (2002); B. B. Back et al., PHOBOS Collaboration, ibid. 87, 102301 (2001).

[25] J. L. Klay, E895 Collaboration, Ph.D. thesis, University of California, Davis, 2001. 
[26] The ratios at low AGS energies were calculated based on the measured midrapidity ratios and assuming that the midrapidity to $4 \pi$ ratio is the same as at $10.74 A \mathrm{GeV}$ [22].

[27] M. Gaździcki and O. Hansen, Nucl. Phys. A528, 754 (1991).

[28] L. D. Landau, Izv. Akad. Nauk SSSR, Ser. Fiz. 17, 51 (1953).

[29] R. Hagedorn, CERN Report CERN-TH-7190-94; in Proceedings of NATO Advanced Study Workshop on Hot Hadronic Matter: Theory and Experiment, Divonne-les-Bains, Switzerland, 1994, edited by J. Letessier, H. Gutbrod, and J. Rafelski [Hot Hadronic Matter 346, 13 (1994)]; J. Cleymans and H. Satz, Z. Phys. C: Part. Fields 57, 135 (1993); J. Sollfrank, M. Gaździcki, U. Heinz, and J. Rafelski, ibid. 61, 659 (1994); P. Braun-Munzinger, J. Stachel, J. Wessels, and N. Xu, Phys. Lett. B 365, 1 (1996): G. D. Yen, M. I. Gorenstein, W. Greiner, and S. N. Yang, Phys. Rev. C 56, 2210 (1997); G. D. Yen and M. I. Gorenstein, ibid. 59, 2788 (1999).
[30] R. Stock, Phys. Lett. B 456, 277 (1999).

[31] J. Cleymans and K. Redlich, Phys. Rev. C 60, 054908 (1999); P. Braun-Munzinger et al., Nucl. Phys. A697, 902 (2002).

[32] F. Becattini, M. Gaździcki, and J. Sollfrank, Eur. Phys. J. C 5, 143 (1998).

[33] H. Sorge, H. Stöcker, and W. Greiner, Nucl. Phys. A498, 567c (1989); H. Sorge, Phys. Rev. C 52, 3291 (1995).

[34] F. Wang, H. Liu, H. Sorge, N. Xu, and J. Yang, Phys. Rev. C 61, 064904 (2000).

[35] S. A. Bass et al., UrQMD Collaboration, Prog. Part. Nucl. Phys. 41, 255 (1998).

[36] H. Weber et al., UrQMD Collaboration, nucl-th/0205030, Phys. Lett. B (to be published).

[37] W. Cassing, E. L. Bratkovskaya, and S. Juchem, Nucl. Phys. A674, 249 (2000). 